\title{
Methods for the Quantification of Resistance of Apple Genotypes to European Fruit Tree Canker Caused by Neonectria ditissima
}

M. Wenneker, Applied Bulbs, Trees \& Fruit Research, Wageningen University \& Research, 6670 AE, Zetten, The Netherlands; P. W.
Goedhart, Biometris, Wageningen University \& Research, 6700 AA, Wageningen, The Netherlands; P. van der Steeg, Applied Bulbs, Trees \&
Fruit Research, Wageningen University \& Research, Zetten; and W. E. van de Weg and H. J. Schouten, ${ }^{\dagger}$ Plant Breeding, Wageningen University \& Research, 6700 AJ, Wageningen, The Netherlands

\begin{abstract}
European fruit tree canker, caused by Neonectria ditissima, is an important disease of pome fruit worldwide. Apple cultivars differ in their levels of susceptibility to $N$. ditissima. In order to design an effective plant resistance test, we examined the effectiveness of two resistance parameters: infection frequency and lesion growth. Both parameters were evaluated in parallel tests using 10 apple cultivars in three experimental years, applying seminatural infection of leaf scars (infection frequency) or inoculation of artificial wounds (lesion growth). We compared six parameters for lesion growth, of which a new parameter, lesion growth rate (LGR), appeared to be the best with respect to reproducibility and statistical

significance. LGR is defined as the slope of the regression of lesion size versus time. The slope was estimated for each lesion, employing a common start date and a lesion-specific end date determined by the girdling of the lesion. The two parameters (infection frequency and LGR) were examined in separate experiments and in three successive years, and provided complementary information and resulted in reproducible conclusions on the relative resistance levels to $N$. ditissima of the tested cultivars. The presented methods can be used to develop strategies for the control of European fruit tree canker (e.g., in the breeding of new apple cultivars with high levels of resistance to $N$. ditissima).
\end{abstract}

European canker is an important disease of pome fruit worldwide, which may cause severe losses mainly in temperate regions (Weber 2014). The disease is caused by the fungal pathogen Neonectria ditissima ( N. galligena, formerly Nectria galligena; anamorph Cylindrocarpon heteronema). The formation of cankers on woody tissue is the most obvious symptom of Neonectria ditissima. Cankers can be formed on twigs, branches, and the main stem (Cooke 1999). Growing cankers often girdle twigs and branches, and eventually kill parts of a tree. When cankers are formed on minor branches, growers will prune them out. However, cankers formed on major branches or on the main stem may lead to the loss of whole trees (McCracken et al. 2003). N. ditissima also causes fruit rotting of apple, which may develop already in the orchard or later, during storage (Berrie 1989; Xu and Robinson 2010).

$N$. ditissima is a wound pathogen, and all kinds of natural and artificial wounds can be infected (Swinburne 1975; Xu et al. 1998). Leaf scars formed during leaf fall are considered to be the major site of infection (Dubin and English 1974). Warm and humid weather with frequent rainfall is favorable for infections (Beresford and Kim 2011). The fungus overwinters as mycelium in twigs and callus tissue of cankers, or as perithecia in cankered wood. Infection may be caused by conidia and ascospores (Swinburne 1975). N. ditissima produces ascospores in perithecia and conidia in sporodochia. Ascospores are released at the end of winter and during spring. On old cankers, conidia and ascospores can be seen year round but their production and germination depend on climate conditions. Rain is an important vector of the pathogen by aerial rain splash from tree to tree and by rain splash and runoff within infected trees (Swinburne 1975). Sporulation, spore dispersal, and infection of N. ditissima are favored by rainfall (McCracken et al. 2003). Frequency, duration of rainfall, and surface wetness are considered to be more important than amount of rainfall (Beresford and Kim 2011; Dubin and English 1974; Latorre et al. 2002; Swinburne 1975; Xu et al. 1998).

${ }^{\dagger}$ Corresponding author: H. J. Schouten; E-mail: Henk.Schouten@wur.nl

*The $\boldsymbol{e}$-Xtra logo stands for "electronic extra" and indicates that three supplementary figures and two supplementary tables are published online.

Accepted for publication 13 July 2017.

() 2017 The American Phytopathological Society
Apple cultivars differ in their level of susceptibility to $N$. ditissima. 'Jonathan' apple is considered to be fairly resistant, 'Elstar' and 'Jonagold' to be moderately to fairly resistant, and 'Kanzi' and 'Gala' to be highly susceptible (Garkava-Gustavsson et al. 2013, 2016; Gómez-Cortecero et al. 2016; Grabowski 1992; Krähmer and Schmidle 1979; Krüger 1983; Palm et al. 2011; Pedersen et al. 1994; van de Weg 1989a,b; van de Weg et al. 1992). However, these reports sometimes disagree in the levels of resistances for these cultivars. Xu et al. (1998) listed a number of causes that might explain these differences. In some studies, canker size was used as a resistance criterion whereas, in other studies, canker incidence or incubation period was recorded. The relationships between canker incidence, incubation period, and canker size may also depend on cultivars and experimental conditions.

The objective of this study was to evaluate the complementarity or redundancy of two components of resistance (infection frequency and lesion growth) and the usefulness of a series of alternative parameters for lesion growth. Infection frequency and lesion size were quantified for a number of commercially available apple cultivars in three experimental years.

\section{Materials and Methods}

Plant material. Ten commercially available apple cultivars differing in levels of resistance to $N$. ditissima were evaluated (i.e., highly susceptible 'Braeburn', 'Discovery', and Gala; moderately susceptible 'Cox Orange Pippin' and 'Topaz'; and moderately to fairly resistant 'Golden Delicious', Elstar, 'Honeycrisp', Jonathan, and 'Santana') (Kemp et al. 1999).

Six experiments were performed at the Experimental Station of Wageningen University \& Research, Randwijk, The Netherlands. They were conducted with apple trees that were in their second growing season after grafting on rootstock M9. Trees were approximately $1.5 \mathrm{~m}$ in height at the time of the start of the experiments. The potted trees were grown outdoors, in 10-liter pots without any training system (unpruned trees), and were fertilized and watered using drip irrigation.

Components of resistance to European canker. Two components of resistance were examined: (i) infection frequency, which represents the resistance to onset of new lesions, and (ii) lesion growth after artificial inoculation of wounded leaf scars. They were examined in separate experiments, here indexed as 1 and 2, respectively, and in three successive seasons-2003 to 2004, 2004 to 
2005, and 2006 to 2007-here referred to as experiments A, B, and $\mathrm{C}$, respectively. Each of the six experiments (A1 to $\mathrm{C} 2$ ) started with new trees.

Infection frequency (natural infections). Experimental design. In the autumn 2003 (experiment A1), 2004 (experiment B1), and 2006 (experiment $\mathrm{C} 1$ ), potted trees of the 10 cultivars were used for the infection frequency experiments. The trees stayed outdoors until the following spring. The experimental design was a randomized complete block design with eight (experiment A1) or four (experiments B1 and C1) blocks, whereby each block contained one tree of each cultivar.

Inoculation. The trees were subjected to a high infection pressure by hanging prunings with sporulating cankers above the trees in October (i.e., before the first leaf drops occurred) and removing them after complete defoliation of the trees in January (A1 and B1) or February $(\mathrm{C} 1)$. These prunings came from various apple cultivars from unsprayed orchards. In experiment A, prunings of Jonagold and 'Schone van Boskoop' were used; in experiment B, from Santana, Topaz, and Schone van Boskoop; and, in experiment $\mathrm{C}$, from 'Alkmene' and Discovery. Prunings from the different cultivars were placed alternating between two neighboring trees.

Assessment of infection frequency. Infection frequency was defined as the percentage of infected leaf scars on the main stem of a tree. In experiment $A 1$, all leaf scars of the main stem of each tree were counted, as well as the number of leaf scars with cankers. This was performed in mid-April 2004 when cankers were still individually visible. The infection frequency of a tree was calculated as the number of infected leaf scars divided by the total number of scars. This was very laborious and, therefore, in the same experiment A1, the percentage of infected scars was also directly scored by a visual assessment. The observed percentage and the visual percentage were highly correlated $\left(R^{2}=0.94\right)$ and therefore, in experiments B1 and $\mathrm{C} 1$, the trees were only visually assessed. This was performed in mid-May, because individual cankers were still visible at that time.

Data analysis. A logistic regression model which employs the binomial distribution was used to analyze the number of cankers on the stem for experiment A1. Overdispersion relative to the binomial distribution was accounted for by inflating the binomial variance with a so-called overdispersion factor (McCullagh and Nelder 1989). In experiments B1 and C1, percentages rather than counts were observed, and these were analyzed with the same statistical model but with binomial totals equal to 100 . Pairwise differences between cultivars were tested at the 5\% significance level after correcting for differences between blocks.

Lesion growth. Experimental design. A randomized block design was used, in which each apple cultivar occurred once per block, employing eight blocks in experiment $\mathrm{A} 2$ and four blocks in experiments B2 and C2.

Preparation of inoculum. The inoculum was obtained from the same collection of cankers that was used for the natural infection experiments. The cankers were placed in plastic bags 24 to $48 \mathrm{~h}$ before inoculation. During this period, sporodochia were produced. On the day of inoculation, the newly formed sporodochia were washed with sterile distilled water and the conidia were collected. The spore suspension was filtered through cheesecloth. Macroconidia density was determined using a hemocytometer and adjusted to $2 \times 10^{5}$ spores $\mathrm{ml}^{-1}$. Germination of macroconidia was assessed by plating $50 \mu \mathrm{l}$ of the spore suspension on water agar for $24 \mathrm{~h}$ of incubation at $20^{\circ} \mathrm{C}$, after which germinated and nongerminated macroconidia were counted.

Inoculation of artificial leaf scar wounds. In autumn, just before leaf fall, the potted trees were transferred to a plot equipped with rainprotected roofing to avoid secondary infection during the course of the experiment. Next, inoculation was performed during the natural leaf fall period on the main stems. The inoculations were performed according to van de Weg (1989 a,b), with several slight modifications. Stems were wounded by removal of a leaf and by cutting away $1 \mathrm{ml}$ from the upper layer of leaf marks together with the corresponding axillary bud using a scalpel. In experiment A2, each tree was inoculated at six positions, numbered from bottom to top, with at least three leaves between the wounds. The two top inoculations in experiment A2 girdled relatively often and quickly; therefore, in experiments B2 and C2, only the lower four positions were used. This implies that, effectively, four inoculation positions at similar heights were used. The inoculation sites were tagged with paint on the reverse side of the inoculation position, in order to facilitate tracing these sites during inoculation and disease assessments. Within $5 \mathrm{~min}$ after wounding, $10 \mu \mathrm{l}$ of the conidial suspension was placed per leaf mark, using an automatic micropipette. After complete absorption of the suspension into the tissue, wounds were covered with white, acid-free petroleum jelly (Vaseline). The Vaseline was removed 4 days after inoculation using tissue paper. After inoculation, the trees were kept in the unheated greenhouse for nearly a year.

It is known that disease incidence and lesion growth may vary between and within experiments, probably because of sensitivity to experimental conditions such as the temperature and humidity at the time of infection and the quality of the inoculum (GarkavaGustavsson et al. 2013, 2016). Therefore, inoculations were spread over multiple days. A further division in time was needed due to the amount of labor, because the performed experiments were part of larger experiments involving hundreds of trees. For experiment A2, inoculations were carried out during 2 days (13 and 19 November 2003), and these dates were more or less randomly distributed over blocks, genotypes, and positions. In experiment B2, eight inoculation days were systematically used: first, the lowest positions were inoculated block by block and, on subsequent days, higher positions were treated. This was done in such a way that all wounds with the same block and position were inoculated on the same day (Table 1). In experiment $C 2$, three inoculation days were used. On the first day, positions 1 and 2 for all trees in blocks 1 and 2 were treated; on the second day, all four positions in blocks 3 and 4 were treated; and, on the third day, positions 3 and 4 in block 1 and 2 were inoculated (Table 1).

Assessment of lesion size. After inoculation, the trees were kept at the same plot with rain-protected roofing for nearly a year. Measurements of lesion sizes began when the first lesions were visible, and were repeated every 4 to 5 weeks until it was no longer possible to take accurate measurements due to lesion coalescence or tree death. In experiment A2, lesion length was measured on 12 occasions (12 February to 20 August 2004); in experiment B2, on 9 occasions (26 January to 21 October 2005); and, in experiment C2, on 5 occasions (29 January to 21 May 2007). When lesions girdled the stem, thus causing death of the stem above the lesion, measurement of that lesion and the lesions above was terminated. Sizes were measured with a digital caliper, accurate to $1 \mathrm{~mm}$. Inoculations that failed were not used in the statistical analysis. In total, six parameters for lesion growth were compared (Fig. 1), as follows:

1. LESION-1: Lesion size (in millimeters) early in spring, when the first girdling lesions were observed.

2. LESION-2: Lesion size (in millimeters) at the end of the experiment

3. AUDPC-1: Area under disease progress curve from 1 January until the time point at which lesion size LESION-1 was assessed.

4. AUDPC-2: AUDPC from 1 January until the end of the experiment.

5. T15 mm (days): The time point (number of days since 1 January) at which the lesion reached the length of $15 \mathrm{~mm}$.

6. LGR: Lesion growth rate (in millimeters per day). The estimated slope of the linear regression line of lesion size versus time, using a single start date specific for each experiment, and a lesion specific end date determined by girdling of the lesion (Fig. 1).

Table 1. Inoculation dates in experiment B (2004) and experiment C (2006)

\begin{tabular}{|c|c|c|c|c|c|c|c|c|}
\hline \multirow[b]{3}{*}{$\operatorname{Pos}^{\mathbf{b}}$} & \multicolumn{8}{|c|}{ Inoculation dates per block ${ }^{\mathbf{a}}$} \\
\hline & \multicolumn{4}{|c|}{ Experiment B (2004) } & \multicolumn{4}{|c|}{ Experiment C (2006) } \\
\hline & 1 & 2 & 3 & 4 & 1 & 2 & 3 & 4 \\
\hline 1 & $3 \mathrm{~N}$ & 4 & 4 & $v$ & 3 & ct & $\mathrm{gv}$ & \\
\hline 2 & $8 \mathrm{Nov}$ & $8 \mathrm{Nov}$ & 9 Nov & & $31 \mathrm{Oct}$ & 31 Oct & $9 \mathrm{Nov}$ & \\
\hline 3 & $10 \mathrm{Nov}$ & $10 \mathrm{Nov}$ & $10 \mathrm{Nov}$ & 11 & $10 \mathrm{Nov}$ & $10 \mathrm{Nov}$ & $9 \mathrm{Nov}$ & \\
\hline 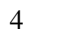 & $11 \mathrm{Nov}$ & $11 \mathrm{Nov}$ & $15 \mathrm{Nov}$ & $15 \mathrm{Nov}$ & $10 \mathrm{Nov}$ & $10 \mathrm{Nov}$ & $9 \mathrm{Nov}$ & 0 \\
\hline
\end{tabular}

${ }^{\mathrm{a}}$ Oct $=$ October and Nov $=$ November

${ }^{\mathrm{b}}$ Inoculation position. 
The AUDPC was calculated using the trapezium rule. The LGR was estimated by the slope of the linear regression line through the data points. The data series used varied between lesions. The start date was common to all lesions of the same experiment and was deduced from the data; it was the last date of observation at which, as yet, no lesion growth was observed. The end date was lesion specific; it was taken as the date at which the lesion stopped growing, resulting in an almost flat part of the lesion growth curve. This date usually coincides with girdling of the stem at the lesion position or a lower position. Individual growth curves for each lesion and the estimated linear regression line are shown in Supplementary Figures $\mathrm{S} 1, \mathrm{~S} 2$, and $\mathrm{S} 3$

Data analysis. The six parameters for lesion size were initially statistically analyzed for each experiment and position separately. Parameters were analyzed according to the randomized block design, implying that genotype differences were tested after correcting for differences between blocks. This was done by means of linear regression rather than analysis of variance because, occasionally, observations were missing, causing unbalance in the design. In all three experiments, trees were inoculated at various dates. In experiments $\mathrm{B} 2$ and $\mathrm{C} 2$, inoculation dates coincided with blocks within each position and, therefore, correcting for blocks also corrected for additive differences in inoculation dates. However, in experiment $\mathrm{A} 2$, the two inoculation dates did not coincide with blocks and, therefore, an additional correction was made for possible differences between inoculation dates. To stabilize the variance, all parameters were $\log$ transformed prior to statistical analysis. Pairwise differences between genotypes were tested at the $5 \%$ significance level.

It was also investigated whether a combined analysis would be possible for the four different lesion positions within each experiment. To this end, Bartlett's test for homogeneity of variance was employed to test whether the four residual variances for each position were similar. This was generally the case and, therefore, a combined analysis of the four positions was employed, still separately for each experiment. The combined analysis was a split-plot analysis in which differences between cultivars were tested at the tree level, whereas lesion position and the interaction between cultivar and position were tested at the position level within trees. In these combined analyses, possible differences between inoculation dates were accounted for. The model was fitted by means of REML, instead of analysis of variance, with 'block/tree/position' as random model and 'inoculationDate + cultivar*position' as fixed model, where date of inoculation is considered a correction factor.
Consistency across lesion positions for a parameter was quantified by examining the two-way table of means classified by cultivar and position, which was obtained from the separate analyses. If the differences between cultivars were identical for each position, we concluded that lesion position was irrelevant and, thus, that the parameter was consistent with respect to position. In that case, an additive regression model with terms cultivar and position, fitted to the two-way table, would give a perfect fit. Thus, discrepancies could be measured by the adjusted $R^{2}$ of the additive model when fitted to the two-way table, where a large $R^{2}$ implies more consistency than a low $R^{2}$. This was done for each experiment separately. In the same way, consistency across experiments could be measured by fitting a model with terms experiment and cultivar to the two-way table of means classified by experiment and cultivar, which was obtained from the three combined analyses. Note that these means are obtained from the combined model without the interaction between positions and cultivar because this interaction was never significant (see Results). By comparing $R^{2}$ values among the six different parameters for lesion size, we were able to select the parameter with the highest consistency.

The parameters for lesion growth were further evaluated for statistical significance (i.e., their ability to reveal significant differences between cultivars). This was assessed by the mean of the $F$ value for statistical testing of differences between cultivar, where the mean is taken over the separate analyses of the four inoculation positions.

\section{Results}

Components of resistance to European canker. Apple cultivars differ in their levels of susceptibility to $N$. ditissima, the causal agent of European canker of apple trees. Canker incidence and canker size are mostly used as components of resistance. Most likely, they represent two different resistance mechanisms. Therefore, we assessed both components in two types of experiments carried out in parallel: the first series for measuring the frequency of infections per main tree stem during natural leaf drop and the second series for measuring lesion growth after artificial inoculation of wounds. For lesion growth, we aimed at finding a summarizing parameter that is not sensitive to artifacts, is consistent across lesion positions and across experiments, and is able to reveal significant differences between apple cultivars according to their well-documented reputation.

Infection frequency. One of the parameters that describes disease incidence is infection frequency, which represents the resistance to onset of new lesions. In three experimental periods, apple cultivars were exposed to sporulating cankers during the natural leaf drop period. The overall mean infection percentage across the tested

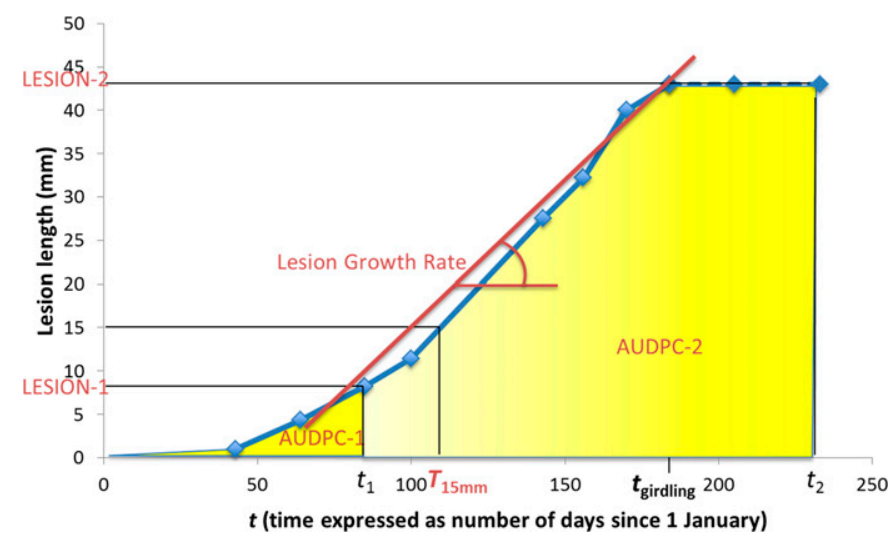

Fig. 1. Six parameters used to characterize lesion growth. The line marked with diamonds represents a typical development of lesion (canker). Observation time points were taken in this example from the growing season of 2004. The six parameters are defined as follows. LESION-1 refers to the lesion size (in millimeters) when very few lesions had caused girdling in the cultivars yet, thus preventing artifacts by girdling. For all genotypes, the same $t_{1}$ value was used within a year but $t_{1}$ varied between years, because the time when girdling started varied between years. LESION-2 refers to lesion size (in millimeters) during the last observation of the year, at $t_{2}$. When girdling by that particular lesion or by a lesion below had occurred earlier in the season, the lesion size was taken just before girdling, as indicated by the dashed line. AUDPC-1 refers to the area under the disease progress curve, starting from the first of January until $t_{1}$. AUDPC-2 is the AUDPC until $t_{2}$, using the dashed line. The parameter T15mm (in days) represents the period since the first of January until the lesion reached the length of $15 \mathrm{~mm}$. Lesion growth rate (millimeters per day) is the slope of the regression line. For estimation of the lesion growth rate, we ignored the lesion sizes after girdling or stem death. 
cultivars was 45,41 , and $52 \%$ for experiments A1, B1, and C1 respectively. Apparently, there was not a predominant effect of the year on the infection frequency.

Cultivar differences in infection percentages. The cultivars showed a wide range in infection percentage (from 8 to 90\%). Significant differences between cultivars were observed (Fig. 2; Supplementary Table S1). Jonathan and Topaz were least affected. In contrast, Gala and Discovery appeared to be highly diseased and, thus, seem to have little resistance to becoming infected. These results were the same for the three experimental periods. The other six cultivars were intermediate and somewhat more variable in their infection frequencies. For instance, Golden Delicious in experiment A1 and Honeycrisp in experiment B1 showed relatively high infection percentages compared with the other experiments and other tested apple cultivars.

Consistency of results from year to year. An important feature of a resistance parameter is consistency of results from year to year. This consistency was generally good: Jonathan and Topaz always had the lowest and second-lowest percentage, respectively, and Discovery and Gala always had the highest infection percentage. The ranking order of the cultivars with intermediate percentages varied among them (Golden Delicious, Santana, Honeycrisp, and Elstar) (Fig. 2). The good consistency is also reflected by the high $R^{2}$ value, which was $75 \%$.

Lesion growth. Lesion growth after infection is the second $N$. ditissima resistance component considered for apple cultivars in our experiments. For the assessment of lesion growth, artificial wounds were made during leaf fall and inoculated with a spore suspension. Necrotic lesions were first observed at 8 to 10 weeks following inoculation. There were no indications of cultivar resistance to infections via artificial inoculation: 96 to $100 \%$ of the inoculations led to growing lesions in all 3 years with our inoculation method. Lesions were similar in appearance to cankers observed in the field. The morphological characteristics of the cankers differed among the cultivars. In the more resistant cultivars, the lesion progressed slowly

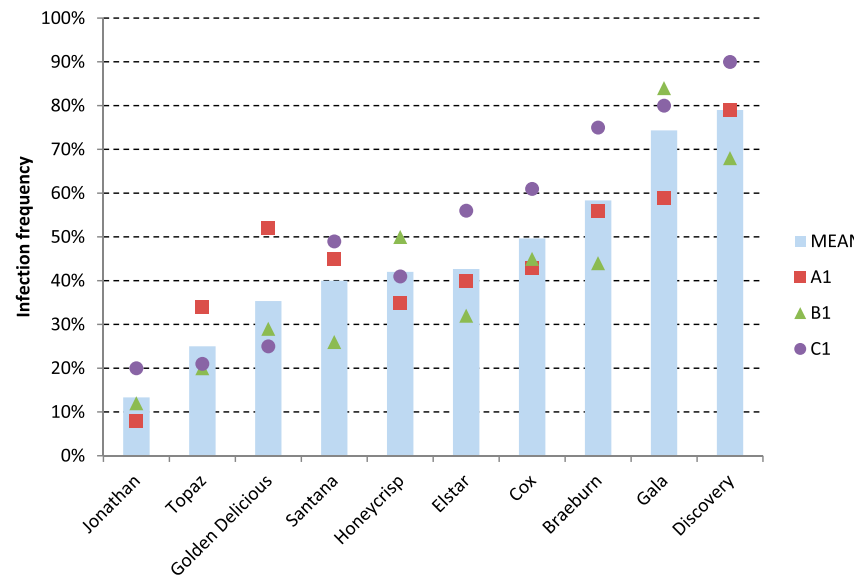

Fig. 2. Percentage of leaf scars showing European canker symptoms due to natural infection on the main stem for the 10 evaluated apple cultivars in three independent experiments (A1, B1, and $C 1)$. and developed as cankers (i.e., callus formation) whereas, in the more susceptible cultivars, the lesions progressed quickly, without signs of callus formation.

In general, the early lesion symptoms consisted of a depressed and discolored area of bark near the point of inoculation. In a later stage of development, lesions progressed primarily vertically across the stem. However, in experiments A2 and C2, lesions frequently girdled the stem, leading to death of the stem above the lesion. The last measurement before the moment of girdling or stem death was used as the final measurement of that lesion.

Parameters for lesion growth. The lengths of the individual lesions were plotted over time. A typical example of lesion growth is depicted in Figure 1. This figure illustrates that factors such as the length of the latency period and girdling of the stem might influence final length of the lesions. For this reason, we evaluated six different parameters for lesion size: LESION-1, LESION-2, AUDPC-1, AUDPC-2, T15 mm, and LGR.

Separate analysis per inoculation position and the combined analysis. The six logarithmically transformed resistance parameters were statistically analyzed separately for each experiment and position. In addition, a combined analysis was performed on the four positions within each experiment, also separately for each experiment. Resulting pairwise differences between cultivars along with simple ranking of the cultivars are given in Supplementary Table S2. The interaction between cultivar and position of inoculation was never significant in the combined analysis, whereas significant differences between positions were found for some parameters in some experiments. This implies that lesion size parameters may be influenced by the position of the inoculation on the main stem but that differences in lesion size parameters between cultivars are similar for all four positions.

Consistency of parameters. Consistency across lesion positions was assessed by means of the adjusted $R^{2}$ of the additive regression model applied to the cultivar by position table of means, separately for each experiment. The parameters LESION-1 and AUDPC-1 had low $R^{2}$ values (Table 2), indicating low consistency, whereas LESION-2, AUDPC-2, T15 mm, and LGR had higher $R^{2}$ values. The growth rate parameter (LGR) had the largest consistency in experiments $\mathrm{A} 2$ and $\mathrm{B} 2$ whereas, in experiment $\mathrm{C} 2$, parameter $\mathrm{T} 15 \mathrm{~mm}$ was more consistent. Overall, LGR had the largest consistency across lesion positions within experiments. Consistency across experiments was also assessed by the adjusted $R^{2}$ value for the cultivar-experiment table of means resulting from the combined analysis of all four positions. The two LESION parameters had the lowest consistency (Table 2) and LGR had the largest consistency.

Statistical significance. The six parameters were further evaluated for their suitability to reveal significant differences between cultivars. The statistical significance of the parameters was assessed by the $F$ values for statistical differences between cultivars. The LGR parameter had the highest statistical significance across all experiments, as well as for each of the single experiments (Table 3). In only one experiment $(\mathrm{C} 2)$, one other parameter (AUDPC-2) performed equally well. Therefore, LGR appears to be the best parameter for revealing statistically significant differences in lesion size among cultivars.

Evaluation of lesion growth of the tested apple cultivars. Because the LGR was the best-performing parameter regarding consistency

Table 2. Consistency (adjusted $R^{2}$ values) for the six lesion growth parameters across inoculation positions, separately for each experiment and across experiments $^{\mathrm{a}}$

\begin{tabular}{|c|c|c|c|c|c|c|}
\hline \multirow[b]{2}{*}{ Experiment } & \multicolumn{6}{|c|}{ Consistency } \\
\hline & LESION-1 & LESION-2 & AUDPC-1 & AUDPC-2 & T15mm & LGR \\
\hline \multicolumn{7}{|c|}{ Across inoculation position } \\
\hline $\mathrm{A} 2$ & 0.35 & 0.75 & 0.24 & 0.79 & 0.82 & 0.85 \\
\hline $\mathrm{B} 2$ & 0.43 & 0.73 & 0.33 & 0.71 & 0.67 & 0.85 \\
\hline $\mathrm{C} 2$ & 0.59 & 0.61 & 0.53 & 0.62 & 0.67 & 0.59 \\
\hline Mean & 0.46 & 0.70 & 0.37 & 0.71 & 0.72 & 0.76 \\
\hline \multicolumn{7}{|c|}{ Across experiments } \\
\hline Combined & 0.45 & 0.46 & 0.73 & 0.71 & 0.68 & 0.81 \\
\hline
\end{tabular}

${ }^{a}$ Larger values indicate higher consistency. 
and statistical significance, we discuss the LGR for the tested cultivars in more detail. The average LGR among the tested cultivars was $0.27,0.13$, and $0.34 \mathrm{~mm} /$ day in experiments $\mathrm{A} 2$, B2, and $\mathrm{C} 2$, respectively, clearly indicating a strong effect of experimental conditions on lesion growth. Many significant differences were found between the cultivars. In general, the largest LGR values were observed for Gala, Discovery, and Braeburn, and the smallest LGR values for Jonathan, Topaz, Santana, Golden Delicious, Elstar, and Honeycrisp (Fig. 3), with intermediate LGR values for Cox Orange Pippin.

Correlations between components of resistance. The correlation between infection frequency and LGR was also investigated (Fig. 4). In all experiments, a positive correlation between LGR and infection frequency was found, with $R^{2}$ values of $0.43,0.50$, and 0.61 for the experiments $\mathrm{A}, \mathrm{B}$, and $\mathrm{C}$, respectively. A higher $R^{2}$ value of 0.77 was obtained when using mean values calculated over the three experiments.

\section{Discussion}

The goal of this research was to find reliable, quantitative parameters for components of resistance in apple to European fruit tree canker caused by the fungal pathogen $N$. ditissima. Important criteria for such parameters are (i) consistency across experiments, (ii) sufficient resolution to reveal genetic differences between apple genotypes, (iii) insensitivity to particular disease-specific artifacts, and (iv) representation of distinctive components of resistance. To this end, we evaluated infection frequency and lesion growth for 10 commercially available apple cultivars and examined six alternative parameters for lesion growth.

In apple, leaf scars that arise during leaf fall in autumn are a main port of entrance for $N$. ditissima. Therefore, our experiments on infection frequency were carried out with naturally formed leaf scars under outdoor weather conditions. In these experiments, we used potted trees that were exposed to inoculum sources (cankered prunings) during the entire leaf drop period. The results obtained over three experimental years showed that the infection frequency as a parameter for resistance to $N$. ditissima was reproducible, with sufficient statistical significance differences between cultivars. However, some

Table 3. Statistical significances: $F$ values for cultivar for the six resistance parameters based on lesion growth ${ }^{\mathrm{a}}$

\begin{tabular}{lcccccc}
\hline Experiment & LESION-1 & LESION-2 & AUDPC-1 & AUDPC-2 & T15mm & LGR \\
\hline A2 & 1.19 & 2.68 & 1.38 & 3.29 & 4.09 & 5.52 \\
B2 & 2.08 & 2.90 & 1.25 & 2.92 & 2.37 & 3.21 \\
C2 & 3.16 & 4.09 & 3.31 & 3.74 & 3.22 & 4.04 \\
Mean & 2.14 & 3.22 & 1.98 & 3.32 & 3.23 & 4.26 \\
\hline
\end{tabular}

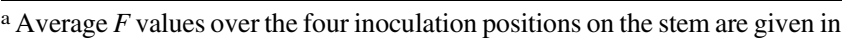
the bottom line. A high $F$ value implies a high statistical significance.

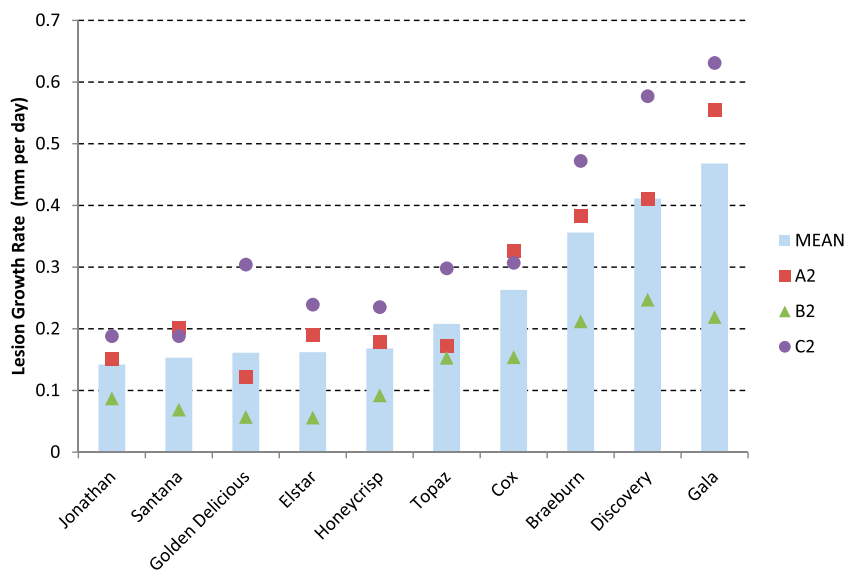

Fig. 3. Lesion growth rate for the 10 evaluated apple cultivars in the three experiments $\mathrm{A} 2$ to $\mathrm{C} 2$. genotypes with an intermediate level of resistance showed variable results over the experimental years. Thus, for a reliable evaluation of the genotypes with intermediate levels of resistance for leaf scar infection, multiple experiments are necessary.

Infection percentage as a parameter for resistance were successfully applied by Alston (1970), Garkava-Gustavsson et al. (2016), and van de Weg (1989a). Our experimental conditions seemed more conducive for infection than those of Garkava-Gustavsson et al. (2016), given that their infection percentages ranged from 0 to $35 \%$ whereas ours ranged from 13 to $79 \%$, despite following similar experimental approaches. Our range was only slightly less wide than those obtained by Alston (1970) (0 to 86\%) and van de Weg (1989a) (3 to $100 \%$ ), who both performed inoculation of artificial wounds. Moreover, classifications of cultivar susceptibility to infection were consistent among all studies for the (few) commonly tested cultivars. Apparently, various experimental approaches can be used to obtain similar information on the relative resistance of apple cultivars to infection.

Under natural conditions, $N$. galligena requires an appropriate wetness period to infect, which is also true for leaf scars (Latorre et al. 2002). Evaluation of leaf drop periods and weather conditions, especially precipitation, revealed that leaf scars of all cultivars were frequently exposed to possible infections from the first leaf drop until the last leaf drop. Also, the majority of cultivars had their main leaf drop during the same period (often after some days of low temperature). This makes it unlikely that specific cultivars escaped from possible $N$. ditissima infections due to weather conditions. Thus, under our conditions, there was no need for water showers (overhead irrigation), as used by Garkava-Gustavsson et al. (2016).

The second parameter we evaluated was lesion growth after inoculation of artificial wounds. In three lesion growth experiments, we successfully aimed for high infection frequencies (close to $100 \%$ ) in order to get a statistically meaningful number of informative lesions with the least possible numbers of trees. This approach was followed in view of cost efficiency and test capacity by maximizing the number of individuals that can be tested within a single experiment, which is of importance for large germplasm screening experiments as for genetic studies on segregating quantitative trait loci mapping populations. We circumvented the need for a wetness period by temporally sealing inoculated wounds with Vaseline. The required moisture probably comes from the wounded plant tissues, including xylem vessels. This approach was shown to be highly efficient because it resulted in infection percentages across cultivars from 97 to $100 \%$ in the current experiments, 95 to $100 \%$ in van de Weg (1989a), and 90\% in van de Weg (1989b).

A complicating factor in measuring lesion size was girdling of inoculated shoots or young stems when the lesions grew wider. As a result of girdling, the lesions stopped expanding, suggesting a final lesion length and, thus, creating a kind of artifact. Moreover, lesions above the site of girdling were affected even more dramatically. In those cases, lesion size measurements are uninformative. Consequently, such values for lesion size will lead to an underestimation of the levels of susceptibility for some cultivars. This is a common problem for this method (Garkava-Gustavsson et al. 2013; Ghasemkhani et al. 2015; Gómez-Cortecero et al. 2016). In view of this problem, these lesions as well as lesions above the one that girdled the stem were given the constant value of the last measurement before girdling by Garkava-Gustavsson et al. (2013), Ghasemkhani et al. (2015), and van de Weg (1989a). To overcome this problem, lesion progress could be estimated by extrapolation but the cultivar-specific patterns of disease progression suggest that such extrapolation would have limited value (Garkava-Gustavsson et al. 2013). Therefore, van de Weg (1989b) ranked cultivars at different stages of disease development; at early stages, the most susceptible cultivars could be distinguished from the less susceptible ones while, at the later stages, highly and moderately resistant genotypes could be distinguished.

In our experiments, we compared six different parameters for quantification of lesion growth (Fig. 1). Of these, LGR appeared to be the best-performing parameter for consistency among lesion positions within a stem (Table 2), for consistency among experiments 

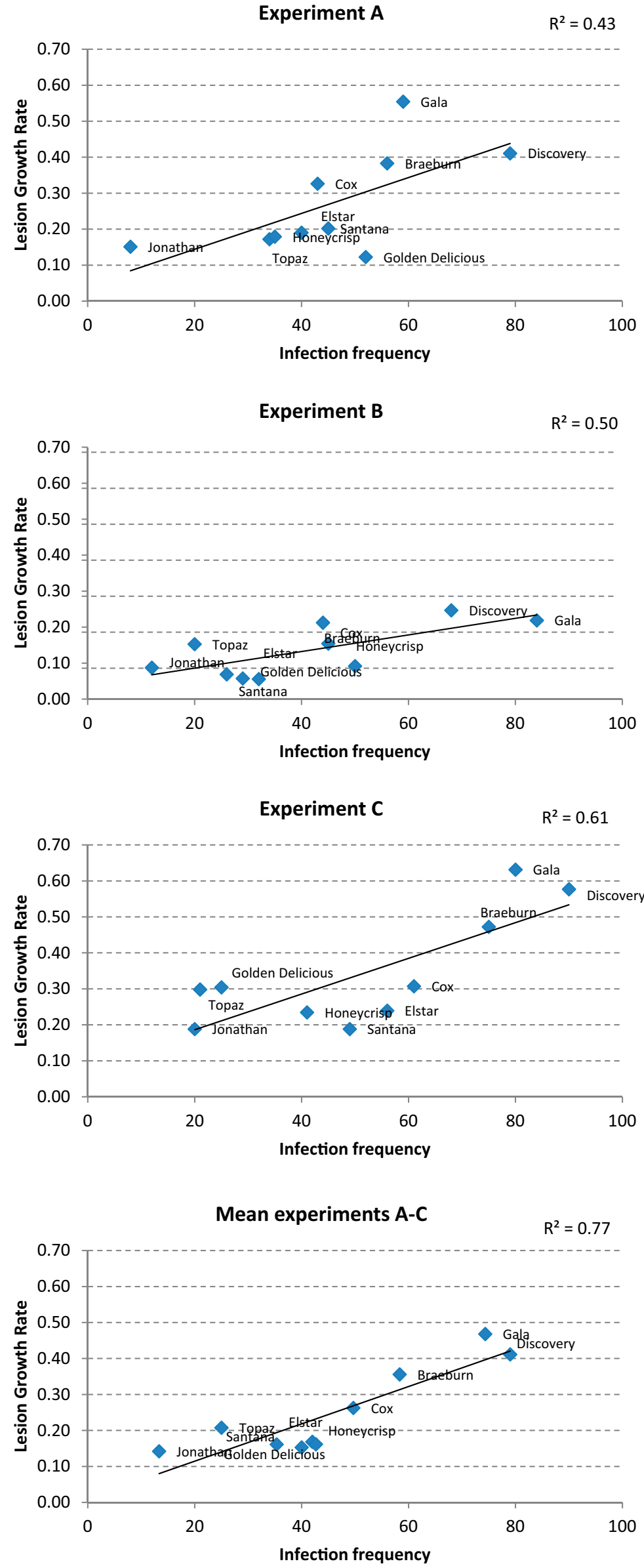

Fig. 4. Relationships between infection frequency and lesion growth rate for the experiments $A, B, C$, and the mean values of $A$ to $C$. 
(Table 2), as well as for statistical significance (Table 3). LGR equals the estimated slope of the regression line of lesion size versus time (Fig. 1). It does not use data after girdling, thus preventing the girdling artifacts that are present in LESION-2 and AUDPC-2. Also, the more resistant individuals have the opportunity to develop lesions for a long time period compared with LESION-1 and AUDPC-1, where disease assessments are halted as soon as the first lesions (usually the most susceptible individuals) girdled the stem.

The advantage of LESION-1 and AUDPC-1 is that the artifact of girdling is prevented. At this time, the lesions of the more resistant individuals are usually still small or even not yet macroscopically visible, thereby possibly giving a poor signal-to-noise ratio and not allowing differentiation between the more resistant individuals. This explains the rather low reproducibility and statistical significance of LESION-1 and AUDPC-1. Further, LGR uses multiple measurements, thus increasing accuracy compared with LESION-1, LESION-2, and T15 mm (which is based on the extrapolation between just two data points). Finally, the possibility to monitor disease development over a relatively long time period gives LGR the ability to reflect the interaction between plant and pathogen across a wider range of physiological and developmental stages and weather conditions. This may give the best prospects to differentiate individuals for their resistance to $N$. ditissima, as observed in the orchard under standard cultural practices. In view of these qualities, we recommend LGR as the parameter of choice.

The LGR values were estimated using linear regression. A prerequisite of LGR is an approximately linear growth of the lesions. This condition of linear growth was generally met in the three experimental years and will generally be the best approach when lesion size is assessed for a number of months rather than weeks.

The mean LGR appeared to vary strongly among the three experimental years $(0.27,0.13$, and $0.34 \mathrm{~mm} /$ day in experiments $\mathrm{A} 2, \mathrm{~B} 2$, and $\mathrm{C} 2$, respectively). The cause for this is not clear and might be due to differences in temperature and relative humidity in the growing season as well as during the first days after inoculation (van de Weg et al. 1992). However, the relative differences in LGR values of resistant and susceptible cultivars appeared to be consistent for the three experiments.

The parameter $\mathrm{T} 15 \mathrm{~mm}$ was an interesting solution for referring to larger lesions for the more resistant individuals while the assessment of the most susceptible individuals is not affected by girdling. Overall, this parameter outperformed LESION-1, AUDPC-1, and LESION-2 with respect to reproducibility among years and statistical significance, and performed similarly to AUDPC-2. Parameter T15 mm also has the advantage that the time needed for disease assessment drops more rapidly during the course of an experiment, because trees can be excluded from further evaluation once the defined size limit has been reached. Thus, parameter T15 $\mathrm{mm}$ may be considered the second best parameter following LGR.

For artificial inoculation, we made four wounds per stem just below the abscission layer of leaves. The lowest wound appeared to lead to the lowest LGR for all cultivars. In view of this, it is advisable to use comparable inoculation positions, counting from the apex of the tree, when comparing genotypes or treatments.

Infected leaf scars need a certain period before symptoms become visible after inoculation and lesion size can be measured. This latency period was used as a parameter for measuring resistance in several cut shoots experiments (Garkava-Gustavsson et al. 2013; Ghasemkhani et al. 2015; van de Weg 1989a). They found latency to be a useful parameter for this type of short-running experiment, where the plant material used would not last for long. However, in these experiments, some cultivars showed only a minor sinking in or blackening of the stem. This could hamper correct assessment of the latency period. Different cultivars also showed different kinds of symptoms, which also complicates assessment of the latency period. Both aspects (i.e., necrosis and variation in symptom type) could be accounted for by the use of a modified latency period, defined as the time needed after inoculation to reach a certain small lesion size such as $5 \mathrm{~mm}$ (W. E. van de Weg, personal communication) In addition, the infection sometimes developed quickly, which required numerous data recordings within a short time window. This could be achievable with small-sized cut-shoot experiments but not with largescale experiments on trees. Moreover, latency period reflects the resistance of an individual colonization rate only for a very short time interval and for a specific stage in the interaction between plant and pathogen, whereas lesion size parameters as used in this research allow long-term evaluations. For these reasons, we did not include latency period in our experiments.

Initial infection and subsequent lesion growth are different stages in disease development in which different components of resistance might be involved. Therefore, we investigated both aspects in independent experiments. Infection frequency and LGR showed quite a high correlation for their mean values of cultivars over the three experimental periods $\left(R^{2}\right.$ of 0.77 ; Fig. 4D). This high correlation was due to small deviations from the regression line along the entire scale of observations rather than the presence of a major group of clustered cultivars and one or two outliers. This implies that the two parameters (i.e., infection frequency and LGR) are associated with one another, although they refer to different phases in the pathogenesis. Practically, this may mean that, when resources and labor are limited, one may choose for one parameter only. The statistical significance for infection frequency was better than for LGR. Apparently, this implies that, with infection frequency as a parameter, it is easier to find significant differences between cultivars. Moreover, this method required far less labor input compared with LGR. Consequently, infection frequency can be regarded as the preferred method compared with lesion growth. However, in view of variability between years and the low correlation within single-year experiments, we recommend that these measurements be repeated during different years. Indeed, single-year results may lead to misleading conclusions with both parameters. For instance, in experiment A1, the low susceptible Golden Delicious was more infected than moderately susceptible 'Cox' (Fig. 4A) whereas, in experiment C2, both cultivars showed the same LGR (Fig. 4C) although they could clearly be distinguished when using the 3-year data (Fig. 4D). The high variability among years might indicate that the lower correlations for the individual experiments were caused by environmental factors (noise) rather than by different resistance mechanisms.

To the best of our knowledge, the relationship between infection percentage and colonization rate (lesion growth) has been studied only once before in independent experiments (Garkava-Gustavsson et al. 2016). In that research, infection percentage and colonization rate (evaluated as AUDPC) seemed to provide complementary information. For example, Gala was second highest for AUDPC but had intermediate infection percentages for both experimental years. These different findings might be due to differences in experimental approaches. Garkava-Gustavsson et al. (2016) mentioned to need for further optimization of their approach for assessing infection percentages.

The methods described in this article for the quantification of resistance of apple genotypes to European fruit tree canker may contribute in developing strategies for the control of European fruit tree canker; for example, breeding of new apple cultivars with high levels of resistance to $N$. ditissima infection would be helpful toward more sustainable apple production.

\section{Acknowledgments}

This study was co-funded by the Ministry of Economic Affairs, and Inova Fruit B.V.

\section{Literature Cited}

Alston, F. H. 1970. Response of apple cultivars to canker, Nectria galligena. Rep E. Malling Res. Stn. 1969:147-148.

Beresford, R. M., and Kim, K. S. 2011. Identification of regional climatic conditions favorable for development of European canker of apple. Phytopathology 101: 135-146.

Berrie, A. M. 1989. Storage rots of apple and pear in South East England 1980-88: Incidence and fungicide resistance. In: Integrated Control of Pome Fruit Diseases. B. K. Gessler, ed. IOBC Bull. Vol. II:229-239.

Cooke, L. R. 1999. The influence of fungicide sprays on infection apple cv Bramley's seedling by Nectria galligena. Eur. J. Plant Pathol. 105:783-790. 
Dubin, H. J., and English, H. 1974. Factors affecting apple leaf scar infection by Nectria galligena conidia. Phytopathology 64:1201-1203.

Garkava-Gustavsson, L., Ghasemkhani, M., Zborowska, L., Englund, J.-E., Lateur, M., and van de Weg, E. 2016. Approaches for evaluation of resistance to European canker (Neonectria ditissima) in apple. Acta Hortic. 1127:75-82.

Garkava-Gustavsson, L., Zborwska, A., Sehic, J., Rur, M., Nybom H., Englund, J.-E., Lateur, M., Weg, Van de Weg, E., and Holefors, A. 2013. Screening of apple cultivars for resistance to European canker, Neonectria ditissima. Acta Hortic. 976:529-536.

Ghasemkhani, M., Liljeroth, E., Zborawska, A., and Nyborn, H. 2015. Cut-off shoots method for estimation of partial resistance in apple cultivars to fruit tree canker caused by Neonectria ditissima. Acta Agric. Scand. Sect. B 65: $412-421$.

Gómez-Cortecero, A., Saville, R. J., Scheper, R. W. A., Bowen, J. K., Agripino De Medeiros, H., Kingsnorth, J., Xu, X., and Harrison, R. J. 2016. Variation in host and pathogen in the Neonectria/Malus interaction; toward an understanding of the genetic basis of resistance to European Canker. Front. Plant Sci. 7:1365

Grabowski, M. 1992. Sensitivity of selected cultivars apple trees to Nectria galligena Bres. infection. Sci. Pap. Agric. U. Krakow 20:83-93.

Kemp, H., Wertheim, S. J., and Roelofs, F. P. 1999. Appel. In: 19de Rassenlijst voor Groot Fruitgewassen 1999. CPRO-DLO, Wageningen, The Netherlands.

Krähmer, H., and Schmidle, A. 1979. Uber die Anfalligkeit neuerer Apfelsorten fur Nectria galligena Bres. and Phytophthora cactorum. Nachrichtenbl. Dtsch. Pflanzenschutzdienst (Berlin)31:89-92.

Krüger, J. 1983. Anfalligkeiten von Apfelsorten and Kreuzungsnachkommenschaften für den Obstbaumkrebs nach naturlicher and künstlicher Infektion. ErwerbsObstbau 25:114-116.

Latorre, B. A., Rioja, M. E., Lillo, C., and Muñoz, M. 2002. The effect of temperature and wetness duration on infection and a warning system for European canker (Nectria galligena) of apple in Chile. Crop Prot. 21: 285-291.
McCracken, A. R., Berrie, A., Barbara, D. J., Locke, T., Cooke, L. R., Phelps, K., Swinburne, T. R., Brown, A. E., Ellerker, B., and Langrell, S. R. H. 2003 Relative significance of nursery infections and orchard inoculum in the development and spread of apple canker (Nectria galligena) in young orchards. Plant Pathol. 52:553-566.

McCullagh, P., and Nelder, J. A. 1989. Generalized Linear Models, 2nd ed. Chapman and Hall, London.

Palm, G., Harms, F., and Vollmer, I. 2011. Mehrjärige Befallsentwicklung des Obstbaukrebses an verschiedenen Apfelsorten an drei Standorten. Mitt Obstbauversuchsringes Alten Landes 66:360-363.

Pedersen, H. L., Christensen, J. V., and Hansen, P. 1994. Susceptibility of 15 apple cultivars to apple scab, powdery mildew, canker and mites. Fruit Var. J. 48: 97-100.

Swinburne, T. R. 1975. European canker of apple (Nectria galligena). Rev. Plant Pathol. 54:787-799.

van de Weg, W. E. 1989a. Screening for resistance to Nectria galligena Bres. in cut shoots of apple. Euphytica 42:233-240.

van de Weg, W. E. 1989b. Screening for resistance to Nectria galligena; differences in resistance between seedling populations. In: Integrated Control of Pome Fruit Diseases. IOBC Bull. Vol. XII:137-145.

van de Weg, W. E., Giezen, S., and Jansen, R. C. 1992. Influence of temperature on infection of seven apple cultivars by Nectria galligena. Acta Phytopathol Entomol. Hung. 27:631-635.

Weber, R. W. S. 2014. Biology and control of the apple canker fungus Neonectria ditissima (syn. N. galligena) from a Northwestern European perspective. Erwerbs-Obstbau 56:95-107.

Xu, X.-M., Butt, D. J., and Ridout, M. S. 1998. The effects of inoculum dose, duration of wet period, temperature and wound age on infection by Nectria galligena of pruning wounds on apple. Eur. J. Plant Pathol. 104:511-519.

Xu, X.-M., and Robinson, J. D. 2010. Effects of fruit maturity and wetness on the infection of apple fruit by Neonectria galligena. Plant Pathol. 59:542-547. 